\title{
Transmission Control Policy Design for Decentralized Detection in Tree Topology Sensor Networks
}

\author{
Ashraf Tantawy, Xenofon Koutsoukos, and Gautam Biswas \\ Institute for Software Integrated Systems (ISIS) and Department of Electrical Engineering and Computer Science \\ Vanderbilt University, Nashville, TN, 37235, USA \\ Email: ashraf.tantawy,xenofon.koutsoukos,gautam.biswas@vanderbilt.edu
}

\begin{abstract}
A Wireless Sensor Network (WSN) deployed for detection applications has the distinguishing feature that sensors cooperate to perform the detection task. Therefore, the decoupled design approach that is typically used to design communication networks, where each network layer is designed independently, does not lead to the desired optimal detection performance. Cross-layer design has been recently explored for the design of MAC protocols for parallel topology (single hop) networks, but little work has been done on the integration of communication and information fusion for tree networks. In this work, we design the optimal Transmission Control Policy (TCP) that coordinates the communication between sensor nodes connected in a tree configuration, in order to optimize the detection performance. We integrate the Quality of Information (QoI), Channel State Information (CSI), and Residual Energy Information (REI) for each sensor into the system model. We formulate a constrained nonlinear optimization problem to find the optimal TCP design variables. We solve the optimization problem using a hierarchical approach where smaller local optimization problems are solved by each parent node to find the optimal TCP design variables for its child nodes. We compare our design with the max throughput and decoupled design approaches.
\end{abstract}

Keywords: Decentralized detection, networked information fusion, transmission control policy, optimization

\section{INTRODUCTION}

The deployment of Wireless Sensor Networks (WSNs) in decentralized detection applications is motivated by the availability of low cost sensors with computational capabilities, combined with the advances in communication network technologies. In Decentralized Detection (DD), multiple sensors collaborate to distinguish between two or more hypotheses. In many practical applications, sensors are distributed geographically and connected in a tree configuration to sample the environment, pre-process the data, and communicate the information to the fusion center for final decision-making. The tree topology is preferred over direct transmission as it saves sensor energy and minimizes the fading channel effect.

The classical problem for DD with the tree configuration is to find the local sensor detection strategies (quantization rules) to minimize a system-wide cost function using different

This work was supported in part by ARO MURI grant W911NF-06-1-0076 and by a Vanderbilt Discovery Grant. channel models [1]. This classical quantization problem is unlikely to play a major rule in modern WSNs. The reason is twofold: 1) performance loss due to quantization decays rapidly with the number of information bits in the packet payload [2], [3], and 2) the payload of a packet could be considered large enough to represent local sensor information with adequate accuracy, as additional bits in the payload are unlikely to affect power or delay, given the relatively large packet overhead [4], [5]. On the other hand, the deployment of WSNs in detection applications brings new challenges to the field. In addition to the design of signal processing algorithms at the application layer that has been previously addressed [6], protocols for other communication layers have to be designed to optimize the detection performance.

The layered approach commonly adopted to design wireless networks may not be appropriate for detection applications. Although the layered approach provides simplicity in the design due to the decoupling of system layers, it neither provides the optimal resource allocation nor exploits the application domain knowledge. As an example, throughput is a common performance metric used to design media access control protocols. In DD applications, maximizing the throughput is not the prime objective, rather, maximizing the quality of the information received that yields the best detection performance is the prime objective. Accordingly, a cross-layer design approach is desired for efficient implementation of WSNs in decentralized detection applications.

The cross-layer design approach has been recently explored for the design of Media Access Control (MAC) protocols for parallel topology (direct transmission) sensor networks in detection applications. Decision fusion over slotted ALOHA MAC employing a collision resolution algorithm is studied in [7]. Identical sensors are considered, and the objective is to analyze the detection performance. A more thorough investigation of the design of MAC transmission policies to minimize the error probability has been considered in [8], where sensors are assumed non-identical, and the MAC policy is assumed stochastic. The approach is also generalized to the multi-cluster networks. The cross-layer approach is also considered in [5] where an integrated model for the physical 
channel and the queuing behavior for sensors is developed. The design problem is to choose the code rate and the number of sensors to minimize the error probability for an FDMA system, where orthogonal channels are used between sensors and the fusion center.

Some work has been done on the integration of communication and information fusion for tree networks. Energyefficient routing for signal detection in WSNs is considered in [9], where the objective is to find the optimal route for local data from a target location to the fusion center, to maximize the detection performance or to minimize the energy consumption. Cooperative routing for distributed detection in large sensor networks is studied in [10] using a link metric that characterizes the detection error exponent. Optimal communication rate allocation for multihop sensor networks deployed for DD is studied in [11], where no medium access contention is assumed. For a survey on the interplay between signal processing and networking in sensor networks, see [12] and the references therein.

We addressed the design of optimal TCP for paralleltopology sensor networks in [17]. In this paper, we extend the work to design the optimal TCP that coordinates the communication between sensor nodes connected in a tree configuration. We integrate the physical layer, MAC layer, and the detection application layer in one unified system model, that captures the Quality of Information (QoI), Channel State Information (CSI), and Residual Energy Information (REI) for each sensor. Our approach is to express the detection performance measure as a function of the parameters of the integrated system model, and solve a constrained optimization problem to obtain the TCP variables that maximize the detection performance.

In making our modeling choices, we are motivated by the desire to develop a system model that captures the basic features of practical sensor networks, while being amenable to analysis. Specifically, we make the following design assumptions: 1) Tree topology. It is a general topology since it encompasses both the serial and parallel topologies for detection networks. 2) Digital transmission. Although uncoded analog transmission is optimal in a sensor network under certain conditions (see, e.g., [13]), digital transmission is still the choice for cost-effective, commercial off-the-shelf deployments of sensor network applications. The digital transmission promotes the ON/OFF channel model, where the packet is either received successfully or discarded. 3) Slotted ALOHA $M A C$. The traditional assumption of a dedicated orthogonal channel between each sensor node and its parent node may not be feasible in practice. Slotted ALOHA multiaccess scheme, on the other hand, has been successfully deployed in practice. We use a simplified version of the slotted ALOHA protocol, ignoring the protocol specifics, to keep the analysis tractable.

We summarize the contributions of our work as follows: 1) Integrated model for the detection system. The model captures the physical channel, MAC protocol, and the detection application models, and their interactions. The model also incorporates the QoI, CSI, and REI measures for each sensor. 2) Design of a complete transmission control policy. We design the TCP for the tree topology rather than the common parallel topology (single-hop network), and for a finite number of sensors, rather than asymptotically. The TCP variables include retransmission probabilities, communication rates, and power allocation across sensors. 3) Enhanced detection performance. We show that the proposed design approach has a significant improvement in the detection performance over the classical decoupled and maximum throughput approaches.

The rest of the paper is organized as follows: Section II presents the problem formulation. Section III explains the system model. Section IV presents the solution of the optimization problem to obtain the optimal TCP design. Section $\mathrm{V}$ briefly explains two classical design approaches to be compared with the proposed design. Section VI presents a numerical example, and the work is concluded in Section VII.

\section{Problem Formulation}

Figure 1 illustrates the detection system architecture, where a set of $N$ wireless sensors, and a fusion center denoted by FC, are arranged in a tree structure, and collaborate to detect the phenomenon of interest. We assume the tree structure is prespecified, possibly based on sensor locations, and therefore the routing problem is not considered. Initially, the fusion center broadcasts a message containing the location of the phenomenon (target, smoke,...etc) to be detected, soliciting information from different sensors. Each sensor responds to its parent with the following information: 1) sensor location, 2) the average signal to noise ratio of the measured phenomenon at the sensor location, and 3) the energy the sensor will devote to the detection process. This information could be obtained in practice as follows: 1) the sensor location could be estimated by different localization methods [14], and is used, along with channel measurement techniques, to estimate the CSI for the sensor. 2) The average signal to noise ratio of the measured phenomenon, representing the QoI, could be estimated by the sensor using the distance between the sensor and the phenomenon location, prior information about the phenomenon measured, and the information of the channel state between the sensor and the phenomenon location. 3) Finally, the energy devoted for the detection process, representing the REI, is estimated by the sensor from the battery charging state and the desired remaining useful life of the sensor.

Two approaches are possible to calculate the optimal transmission control policy. The global approach, where the fusion center receives the information from all sensors (through their respective parents), calculates the optimal transmission control policy for each sensor by solving a constrained nonlinear optimization problem, and transmits the values of the TCP variables back to the relevant sensors. This global approach may not be feasible in large sensor networks as it is not scalable with the network size, in addition to the fact that the design parameters have to be propagated back from the fusion center down to all network nodes. A more practical approach is the local approach, where each parent node solves a smaller local optimization problem to specify the locally optimal TCP variables for its child nodes. 


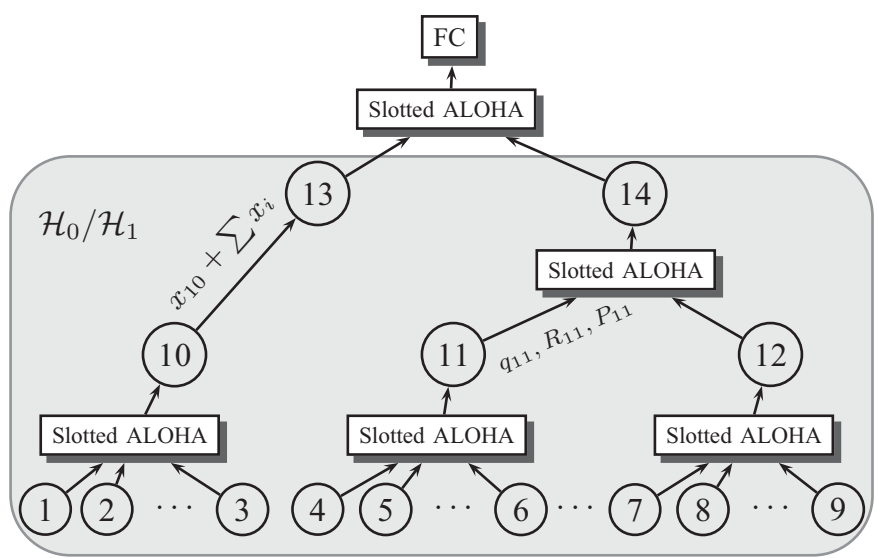

Figure 1. Detection architecture for a tree-topology WSN. Sensors communicate their observations in a multihop fashion over slotted ALOHA multiaccess channels to the fusion center.

Some sensors may not contribute to the detection process, due to either low quality of information (e.g. phenomenon is too far), low channel state (e.g. high noise or long distance to the parent node), or not enough energy to transmit to the parent node (e.g. not enough battery power or long distance to the parent node combined with bad channel quality). The fusion center (global approach) or the parent nodes (local approach) transmit the TCP variables only to the sensors which are specified by the optimization algorithm to be reliable to contribute to the detection task. The resulting values of the TCP variables remain valid for the given location as long as the quality measures for each sensor did not change from the last run of the optimization algorithm.

After each sensor receives the optimal values of the TCP variables, the detection process proceeds as follows: the fusion center broadcasts a message to initiate a detection cycle at the local wireless sensors. Each local sensor samples the environment by collecting a number of observations, and then forms a data packet and communicates its message to the parent node over a shared wireless link using the slotted ALOHA multiaccess control scheme. Parent nodes relay the information of the child nodes, in addition to their own information, through the tree network until reaching the fusion center. Finally, the fusion center makes a final decision after a fixed amount of time representing the maximum allowed delay for detection.

Our focus in this paper is different from the classical decentralized detection literature. We do not treat the problem of optimal local quantization rules at each sensor. Rather, we focus on the problem of optimal network design for detection applications. To obtain a tractable solution, we assume that observations are sent to the fusion center directly without in-network processing. However, the analysis presented here is also applicable when considering in-network compression, although the resultant objective function may result in more complex analysis. In general, since in-network processing results in information loss, while saving network bandwidth and sensor energy, our work could be regarded as an upper bound on the detection performance and a lower bound on the network performance.

\section{System MOdEL}

The detection scheme described above suggests a layered approach to system modeling, as depicted in Figure 2. The physical layer represents the wireless channel model, and defines system parameters such as the communication bit rate and the energy consumed in communicating sensor information to the fusion center. The Media Access Control (MAC) layer represents the slotted ALOHA protocol model, and defines the protocol-specific parameters such as the transmission probability. Finally, the application layer represents the sensing model, and defines the model of the observations obtained by local sensors.

\section{A. Wireless Channel Model}

We present a model for the wireless channel between each parent-child pair in the tree detection network. We focus on the case where the sensor nodes and the fusion center have minimal movement and the environment changes slowly. Since detection applications typically have low communication rate requirements, the coherence time of the wireless channel could be considered much larger than the transmission frame length. Accordingly, only the slow fading component of the wireless channel is considered. Figure 3 shows the fading channel model, where $w(t)$ is an additive white Gaussian noise with power spectral density $N_{0} / 2$. The term $m(d)$ represents the mean path attenuation for a sensor node at a distance $d$ from its parent, where the dependence on time $t$ is dropped since slow fading is considered. We use the Hata path-loss model for the mean path attenuation, where the total $\mathrm{dB}$ power loss is given by [15]:

$$
P_{L}=\underbrace{20 \log _{10}\left(\frac{4 \pi d_{0}}{\lambda}\right)+10 \rho_{c} \log _{10}\left(d / d_{0}\right)}_{\mu_{c}}+X_{\sigma_{c}}
$$

where $d_{0}$ is a reference distance corresponding to a point located in the far field of the transmit antenna, $\lambda$ is the wavelength of the propagating signal, $\rho_{c}$ is the path loss exponent, $d$ is the distance between the transmitting and receiving antennas (i.e. child and parent nodes), and $X_{\sigma_{c}}$ is a zero-mean Gaussian random variable with variance $\sigma_{c}^{2}$. The power loss (in $\mathrm{dB}$ ) is therefore a Gaussian random variable with mean $\mu_{c}$ and variance $\sigma_{c}^{2}$, i.e. $P_{L} \sim \mathcal{N}\left(\mu_{c}, \sigma_{c}^{2}\right)$.

The given wireless channel represents an unreliable bit pipe for the data link layer, with instantaneous Shannon capacity $C=W \log _{2}\left(1+\frac{P_{r}}{N_{0} W}\right) \quad$ bps, where $W$ is the channel bandwidth and $P_{r}$ is the signal power received by the parent node. Using Shannon coding theorem, the data link layer could

\begin{tabular}{|c|c|}
\hline$\underline{\text { Layer }}$ & $\underline{\text { Model }}$ \\
\hline Application & Sensing model \\
\hline MAC & Slotted ALOHA \\
\hline Physical & Fading wireless channel \\
\hline
\end{tabular}

Figure 2. A layered approach to detection system modeling. 


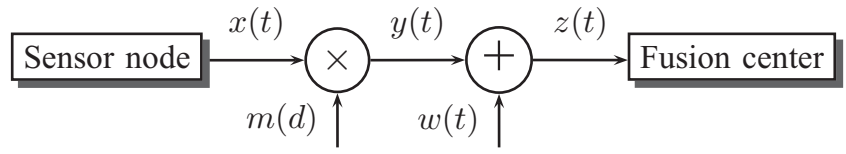

Figure 3. Block diagram for the wireless communication channel. The transmitted signal is subject to large-scale fading and additive white Gaussian noise.

achieve arbitrary communication rates $R$ up to the channel capacity using appropriate coding schemes. Given the state of the art coding schemes that approach the Shannon capacity, we can approximately assume that the fusion center can perform error-free decoding for any transmission with bit rate $R<C$. Therefore, the channel is considered "ON" when $R<C$ and "OFF" otherwise, giving rise to the two-state channel model akin to the one presented in [5]. Noting that $P_{r}=P_{t} 10^{-P_{L} / 10}$, where $P_{t}$ is the average signal power transmitted by the local sensor, and using the result that $P_{L} \sim \mathcal{N}\left(\mu_{c}, \sigma_{c}^{2}\right)$, we get the probability for the channel being "ON" during a transmission:

$$
P[\mathrm{ON}]=\lambda_{c}=\Phi\left[\frac{1}{\sigma_{c}}\left(10 \log \frac{P_{t}}{N_{0} W\left(2^{\frac{R}{W}}-1\right)}-\mu_{c}\right)\right]
$$

where $\Phi($.$) is the cumulative distribution function for the stan-$ dard normal PDF. We note that the Channel State Information (CSI) relevant to our model is represented by the statistics $\sigma_{c}, \mu_{c}$, and $N_{0}$.

\section{B. Media Access Control Protocol Model}

We assume a slotted ALOHA multiaccess communication protocol between each parent node and its child nodes, where each packet requires one time slot for the transmission, all time slots have the same length, and all transmitters are synchronized. Furthermore, we assume simultaneous transmission of parent and child nodes, which could be achieved in practice by sending and receiving on different frequency bands. Finally, subtrees that do not have a common parent (excluding the root node, i.e. the fusion center) do not interfere.

The detection cycle, demonstrated in Figure 4, has length $\tau$, which defines the delay for detection. The detection cycle is divided into a number of transmission slots $L_{i}$, for nodes at the same depth $i$ of the tree, and sharing a common parent. The relationship between the number of slots for consecutive depths is given by $L_{i+1}=m_{i} L_{i}$, where $m_{i}$ is a positive integer. In the following discussion, we designate the set of all child nodes for sensor $k$ by $\mathcal{C}_{k}$, and the set of all siblings (excluding sensor $k$ ) by $\mathcal{B}_{k}$.

At the beginning of every time slot, each local sensor $k$ collects a number of observations $n_{k}$ and forms an information packet for transmission over the wireless channel. The sensor then attempts to transmit to its parent with probability $q_{k}$, transmission power $P_{k}$, and communication rate $R_{k}$. The sensor attempts transmission at each time slot, despite the status of its previous transmission attempts. The final decision

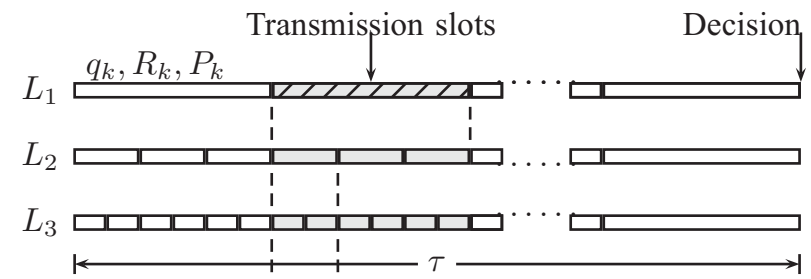

Figure 4. Detection cycle of length $\tau$ is divided into $L_{1}$ slots for nodes at depth 1 that have the same parent, $L_{2}$ slots for nodes at depth 2, and so on. The number of slots at each depth is an integer multiple of the number of slots at the previous depth. Inside each slot, each sensor attempts transmission with the indicated TCP parameters.

is taken at the fusion center using the information received during the detection cycle. The process repeats for every detection request initiated by the fusion center.

The communication rate could be expressed with the aid of Figure (5) as follows:

$$
R_{k}=\frac{b L_{i} n_{k}}{\tau}+\frac{1}{m_{i}} \sum_{v \in \mathcal{C}_{k}} Z_{v} R_{v}, \sum_{v \in \mathcal{C}_{k}} Z_{v}=m_{i}
$$

where $b$ is the number of encoding bits for each observation, $i$ is the depth of the sensor, and $Z_{v}$ is the number of times the child sensor $v$ successfully transmitted during the $m_{i}$ time slots. The first term in (3) represents the information collected by the sensor node, and vanishes if the node functions as a relay node for its child nodes. The second term represents the information received from the child nodes and vanishes for leaf nodes.

We note that in the above description for the MAC protocol, we ignored the acknowledgement slots and any protocol specifics required for synchronization or rate negotiation to simplify the analysis. Also, we ignored the packet overhead, which is a reasonable approximation for practical WSN protocols with large packet payload.

Now, we calculate the overall probability of a successful packet transmission, including the wireless channel effect. We note from (3) that the communication rate of intermediate nodes is a random variable, being dependent on the information received from its child nodes. Accordingly, accurate formulation for the problem requires modification of (2) to include the randomness of the communication rate. Unfortunately, no closed form solution could be obtained

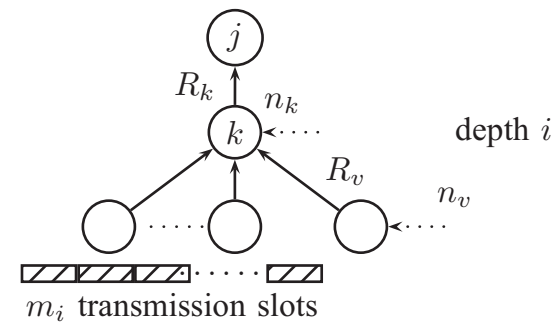

Figure 5. The communication rate for node $k$ is the sum of the communication rate required to send its local observations and the communication rates of its child nodes that successfully transmitted during the previous $m_{i}$ time slots. 
for the channel ON probability in this case. Even If we approximated the sum in the second term of (3) by a Gaussian random variable using the Central Limit Theorem, and used the approximation of $R$ to derive the new probability of success, we would obtain, after some manipulations, a sum of two Log Normal random variables, which does not have a closed-form probability distribution. Therefore, to keep the analysis tractable, we resort to a suboptimal solution, where the communication rate for each node is represented by its expected value. Accordingly, equation (2) is still applicable, where $R$ represents the average communication rate. Now, at any given time slot, the probability of a single packet transmission by sensor $k$ is given by $q_{k} \prod_{v \in \mathcal{B}_{k}}\left(1-q_{v}\right)$. Further, this packet will be successfully received by the parent node if the state of the physical channel between the child node $k$ and the parent node is "ON" during this time slot. Therefore, using (2), the total probability of a successful packet transmission by sensor $k$ is given by:

$$
\lambda_{k}=q_{k}\left[\prod_{v \in \mathcal{B}_{k}}\left(1-q_{v}\right)\right] \Phi\left[\frac{10}{\sigma_{c}^{k}} \log \frac{P_{t}^{k}}{N_{0} W\left(2^{\frac{\bar{R}_{k}}{W}}-1\right)}-\frac{\mu_{c}^{k}}{\sigma_{c}^{k}}\right]
$$

where $\bar{R}_{k}$ is obtained by taking the expected value for (3) and noting that $Z_{v}$ is a binomially distributed random variable with $E\left[Z_{v}\right]=m_{i} \lambda_{v}$

$$
\bar{R}_{k}=\frac{b L_{i} n_{k}}{\tau}+\sum_{v \in \mathcal{C}_{k}} \lambda_{v} \bar{R}_{v}
$$

\section{Energy Model}

To formulate the energy model for each sensor, we first introduce the definition for the network lifetime. The network lifetime $\mathcal{L}$ could be defined as the average time span from the deployment to the instant when the network can no longer perform the task [12]. The network lifetime could be expressed as:

$$
\mathcal{L}=\frac{\mathcal{E}^{0}-\mathcal{E}^{w}}{f_{r} \mathcal{E}^{r}}
$$

where $\mathcal{E}^{0}=\sum_{i=1}^{N} e_{i}^{0}$ is the total initial energy in all sensors at the time of deployment, $\mathcal{E}_{w}=\sum_{i=1}^{N} e_{i}^{w}$ is the total wasted energy remaining in sensor nodes when the network cannot perform the assigned task, $f_{r}$ is the average sensor reporting rate defined here as the number of detection cycles per unit time, and $\mathcal{E}^{r}=\sum_{i=1}^{N} e_{i}^{r}$ is the expected energy consumed by all sensors in one detection cycle. The total wasted energy could be defined for our detection problem as the energy required to achieve a minimum pre-specified value for the detection performance measure.

In general, we can include the energy allocation problem in our formulation, i.e. finding optimal $e_{i}^{r}$ values for all sensors that maximize the detection performance while guaranteeing a minimum network lifetime. In this work, however, we focus on the optimal TCP problem, and therefore we resort to a simpler energy formulation. First, we assume that $e_{i}^{w}$ is the energy remaining in the sensor battery when the sensor is not capable of operating its electronic circuits for computations and communication, which is fixed and known for each sensor. Second, we assume that the reporting energy for each sensor $e_{i}^{r}$ is a fixed percentage of its net useful energy at the time of sensor deployment. Using these two assumptions, we get the following expression for the energy consumed by each sensor in one detection cycle:

$$
e_{k}^{r}=\frac{e_{k}^{0}-e_{k}^{w}}{f_{r} \mathcal{L}} \quad \forall k
$$

which could be calculated for any desired network lifetime $\mathcal{L}$. The total energy consumed by each sensor is divided between transmission and reception (except for leaf nodes). By assuming that the energy consumed in the reception process is proportional to the detection cycle lifetime with proportionality constant $\alpha$, and by noting that the expected number of transmissions by sensor $k$ during a detection cycle is $L_{i} q_{k}$, we get:

$$
P_{t}^{k}=\frac{\left(e_{k}^{r} / \tau\right)-\alpha}{q_{k}}=\frac{1}{q_{k}}\left(p_{k}-\alpha\right)
$$

where $p_{k}$ is the average transmission power over one detection cycle, which summarizes the Residual Energy Information (REI) for each sensor. Using (8) in (4), we get:

$$
\lambda_{k}=q_{k}\left[\prod_{v \in \mathcal{B}_{k}}\left(1-q_{v}\right)\right] \Phi\left[a_{k}-\left(\frac{10}{\sigma_{c}^{k}}\right) \log q_{k}\left(2^{\frac{\bar{R}_{k}}{W}}-1\right)\right]
$$

where $a_{k}=\frac{1}{\sigma_{c}^{k}}\left(10 \log \frac{p_{k}-\alpha}{N_{0} W}-\mu_{c}^{k}\right)$. We note that $\alpha<p_{k}=$ $e_{k}^{r} / \tau$ for the sensor to be able to transmit the information. In addition, $\alpha=0$ for leaf nodes.

\section{Sensing Model}

We consider a detection application where a set of sensors are randomly placed in a surveillance area to detect the presence of an object. Sensors have fixed positions, which could be estimated using different localization algorithms. The surveillance area is divided into a number of range resolution cells that are probed by local sensors upon receiving a command from the fusion center. We focus our work on detection using signal amplitude measurements. Therefore, when there is an object at a specific resolution cell, the observation at sensor $k$, located at $d_{k}$ distance from the object, could be expressed as:

$$
x_{k}=\frac{\epsilon}{d_{k}^{\eta / 2}}+w_{k}
$$

where $\epsilon$ is the amplitude of the emitted signal at the object, $\eta$ is a known attenuation coefficient, typically between 2 and 4, and $w_{k}$ is an additive white Gaussian noise with zero mean and variance $\sigma_{s}^{k^{2}}$.

The detection problem could be defined as the following binary hypothesis testing problem, for each time slot $i$ :

$$
\begin{aligned}
\mathcal{H}_{0}: x_{k}[j, i] & =w_{k}[j, i] & j & =1,2, \ldots, n_{k} \\
\mathcal{H}_{1}: x_{k}[j, i] & =\mu^{k}+w_{k}[j, i] & j & =1,2, \ldots, n_{k}
\end{aligned}
$$


where $\mu^{k}=\epsilon / d_{k}^{\eta / 2}$, and $n_{k}$ is the number of observations obtained by sensor $k$ at each time slot. We note that noise samples are independent across sensors, i.e., the observations at local sensors are independent across time and space, but not necessarily identically distributed since some sensors may be closer to the measured phenomenon, and noise variances are assumed unequal. We present the following two propositions, without proof, due to space limitations.

Proposition 1. The optimal test statistic at the fusion center for the given system description is given by (13), where $r_{v_{j}}^{i_{1} \ldots i_{j}}$ is a Bernoulli distributed random variable taking the values 0 or 1, with success probability as in (9), representing the success or failure of receiving a packet from sensor $v_{j}$ (located at depth $j$ ) in the slot sequence $i_{1} \ldots i_{j}$ (slot $i_{1}$ is divided into $m_{1} i_{2}$ slots, which is divided into $m_{2} i_{3}$ slots, and so on). $l$ represents the depth of the tree, and $x_{v}\left[j, i_{1} \ldots i_{l}\right]$ represents observation number $j$ received from sensor $v$ in the slot sequence $i_{1} \ldots i_{l}$.

The expression in (13) is simply a weighted sum of the observations received at the fusion center. The complexity of the equation comes from the fact that successful reception of the observations of child nodes at the fusion center depends on the success of the transmission of all parent nodes up to the fusion center.

We adopt the deflection coefficient as a detection performance measure, defined as [16]:

$$
d^{2}=\frac{\left(E\left[V ; \mathcal{H}_{1}\right]-E\left[V ; \mathcal{H}_{0}\right]\right)^{2}}{\operatorname{var}\left[V ; \mathcal{H}_{0}\right]}
$$

which provides more tractable results in our study. The deflection coefficient is also closely related to other performance measures, e.g., Receiver Operating Characteristics (ROC) curve. In general, the detection performance monotonically increases with increasing the deflection coefficient.

Proposition 2. The deflection coefficient for the detector in (13) is given by (14), where $c_{v}=\left(\mu^{v} / \sigma_{s}^{v}\right)^{2}$.

We define the deflection coefficient for individual sensors as $d_{v}^{2}=n_{v} c_{v}$ and adopt it as a measure of its Quality of Information (QoI). Using (5) in (14), we obtain our objective function in (15).

Table I lists the model parameters and their description. The third column classifies each parameter according to its method of calculation as either given from the application knowledge, estimated online, calculated, or as a design parameter. The fourth column highlights the parameters that are a measure of the REI, CSI, or QoI for each sensor. The last column classifies each parameter according to its relevant layer in the system model.

\section{TCP Design For Optimal Detection}

The optimization problem could be summarized as follows:

$$
\max _{\boldsymbol{q}, \overline{\boldsymbol{R}}} d^{2} \quad \text { s.t. } 0 \leq q_{i} \leq 1, \bar{R}_{i} \geq \sum_{v \in \mathcal{C}_{i}} \lambda_{v} \bar{R}_{v} \quad i=1: N
$$

Table I

\begin{tabular}{|c|c|c|c|c|}
\hline Param. & Description & Calculation & Notes & Layer \\
\hline$W$ & Channel bandwidth & $\mathrm{G}$ & & \multirow{6}{*}{ 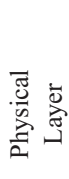 } \\
\hline$N_{0}$ & Power spectral density & E & CSI & \\
\hline$\mu_{c}$ & Mean path loss & $\mathrm{C}(1)$ & CSI & \\
\hline$\sigma_{c}^{2}$ & Path loss variance & E & CSI & \\
\hline$P_{t}$ & Transmission power & $\mathrm{D}$ & & \\
\hline$R$ & Communication bit rate & $\mathrm{D}$ & & \\
\hline$L$ & Number of comm. slots & C (3) & & \multirow{3}{*}{ 岕 } \\
\hline$b$ & Number of encoding bits & $\mathrm{G}$ & & \\
\hline$q$ & Retransmission probability & $\mathrm{D}$ & & \\
\hline$\tau$ & Delay for detection & $\mathrm{G}$ & & \multirow{4}{*}{ 安 } \\
\hline$n$ & Number of observations & $\mathrm{C}(3)$ & & \\
\hline$c$ & Signal to noise ratio & $\mathrm{G}$ & QoI & \\
\hline$e$ & Energy/detection cycle & G & REI & \\
\hline
\end{tabular}

Model Parameters

E: Estimated, G: Given, C (): Calculated (eq. number), D: Design

Although this problem could be solved by existing algorithms (e.g. interior point method) for a local maximum, we note that the objective function in (15) gets more complicated as the tree depth increases. Adding the fact that all design variables have to be propagated back to tree nodes, a more practical approach is clearly needed. If we look at the objective function expression in (15), we note that it reflects the tree hierarchy, i.e. the last term in the expression represents the contribution of the leaf nodes, preceded by the contribution of the parents of the leaf nodes, and so on, until reaching the sensor nodes at the top level of the tree (depth=1). This could be shown by expressing the objective function using the following recursive equation:

$$
d^{2}=\frac{\tau}{b} J_{F C}, \quad J_{k}=\sum_{v \in \mathcal{C}_{k}} \lambda_{v}\left[\bar{R}_{v}\left(c_{v}-c_{k}\right)+J_{v}\right]
$$

where $J_{k}=0$ for leaf nodes and $c_{k}=0$ for the fusion center node. This structure of the objective function suggests a local optimization approach for the problem, where we start by optimizing $J_{v}$ for sensors at depth $l-1$ and continue the local optimization recursively using (17), until reaching the fusion center. This approach is practical since the solution of each local optimization problem could be carried out locally at each parent node. The solution approach is illustrated in Figure (6).

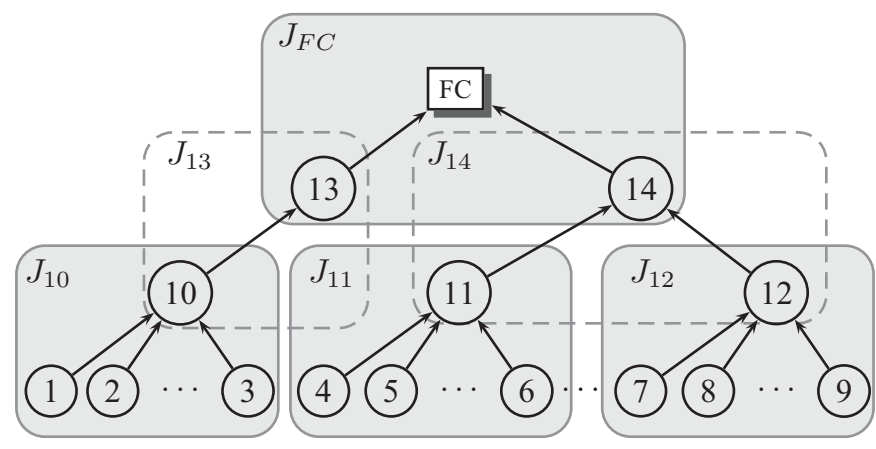

Figure 6. Hierarchical optimization for the TCP design problem. Subtrees are locally optimized starting from the leaf nodes. 


$$
\begin{aligned}
& V=\sum_{i_{1}=1}^{L_{1}} \sum_{v_{1} \in \mathcal{C}_{f}} \sum_{j_{1}=1}^{n_{v_{1}}} r_{v_{1}}^{i_{1}}\left[\left(\frac{\mu^{v_{1}}}{\left.\sigma_{s}^{v^{2}}\right)^{2}}\right) x_{v_{1}}\left[j_{1}, i_{1}\right]+\sum_{i_{2}=1}^{m_{1}} \sum_{v_{2} \in \mathcal{C}_{v_{1}}} \sum_{j_{2}=1}^{n_{v_{2}}} r_{v_{2}}^{i_{1} i_{2}}\left[\left(\frac{\mu^{v_{2}}}{\sigma_{s}^{v_{2}^{2}}}\right) x_{v_{2}}\left[j_{2}, i_{1} i_{2}\right]+\ldots+\sum_{i_{l}=1}^{m_{l}-1} \sum_{v_{l} \in \mathcal{C}_{v_{l}-1}} \sum_{j_{l}=1}^{n_{v_{l}}} r_{v_{l}}^{i_{1} \ldots i_{l}}\left(\frac{\mu^{v_{l}}}{\sigma_{s}^{v_{l}}{ }^{2}}\right) x_{v_{l}}\left[j_{l}, i_{l} i_{2} \ldots i_{l}\right]\right] \ldots\right] \\
& d^{2}=L_{1} \sum_{v_{1} \in \mathcal{C}_{f}} \lambda_{v_{1}}\left[n_{v_{1}} c_{v_{1}}+m_{1} \sum_{v_{2} \in \mathcal{C}_{v_{1}}} \lambda_{v_{2}}\left[n_{v_{2}} c_{v_{2}}+\ldots+m_{d-1} \sum_{v_{l} \in \mathcal{C}_{v_{l-1}}} \lambda n_{v_{l}} c_{v_{l}}\right] \ldots\right] \\
& d^{2}=\frac{\tau}{b} \sum_{v_{1} \in \mathcal{C}_{f}} \lambda_{v_{1}}\left[\bar{R}_{v_{1}} c_{v_{1}}+\sum_{v_{2} \in \mathcal{C}_{v_{1}}} \lambda_{v_{2}}\left[\bar{R}_{v_{2}}\left(c_{v_{2}}-c_{v_{1}}\right)+\sum_{v_{3} \in \mathcal{C}_{v_{3}}} \lambda_{v_{3}}\left[\bar{R}_{v_{3}}\left(c_{v_{3}}-c_{v_{2}}\right)+\ldots+\sum_{v_{d} \in \mathcal{C}_{v_{d-1}}} \lambda_{v_{d}} \bar{R}_{v_{d}}\left(c_{v_{d}}-c_{v_{d-1}}\right)\right]\right] \ldots\right]
\end{aligned}
$$

By substituting (9) in (17), we can express the local optimization problem at parent node $k$ as follows:

$$
\begin{aligned}
& \max \sum_{i \in \mathcal{C}_{k}} q_{i} {\left[\prod_{j \in \mathcal{B}_{i}}\left(1-q_{j}\right)\right]\left[\bar{R}_{i}\left(c_{i}-c_{k}\right)+J_{i}\right] } \\
& \times \Phi\left[a_{i}-\left(\frac{10}{\sigma_{c}^{i}}\right) \log q_{i}\left(2^{\frac{\bar{R}_{i}}{W}}-1\right)\right] \\
& \text { s.t. } 0 \leq q_{i} \leq 1, \quad \bar{R}_{i} \geq \sum_{v \in \mathcal{C}_{i}} \lambda_{v} \bar{R}_{v}=r_{i}
\end{aligned}
$$

We note that $J_{i}$ and $r_{i}$ are fixed values, obtained from solving the local optimization problems at lower levels in the hierarchy. Let the number of child nodes for sensor $k$ is $N_{k}$, and denote the decision variables by $\boldsymbol{x}=\left[\begin{array}{llllllll}q_{1} & q_{2} & \ldots & q_{N_{k}} & R_{1} & R_{2} & \ldots & R_{N_{k}}\end{array}\right]$, where $\boldsymbol{x} \in$ $\mathbb{R}^{2 N_{k}}$, and the objective function by $J(\boldsymbol{x})$, then the optimization problem could be rewritten on the form:

$$
\min _{\boldsymbol{x}}-J(\boldsymbol{x}) \quad \text { subject to } A \boldsymbol{x} \geq \boldsymbol{b}
$$

where

$$
A=\left[\begin{array}{ccc}
I & -I & \mathbf{0} \\
\mathbf{0} & \mathbf{0} & I
\end{array}\right]^{T}, \quad \boldsymbol{b}=\left[\begin{array}{ccc}
\mathbf{0} & \mathbf{- 1} & \boldsymbol{r}
\end{array}\right]^{T}
$$

$I$ is the identity matrix, $\mathbf{0}(\mathbf{1})$ is the vector/matrix of all zeros (ones) with appropriate dimensions, and $\boldsymbol{r}=$ $\left[\begin{array}{llll}r_{1} & r_{2} & \ldots & r_{N_{k}}\end{array}\right]^{T}$. We have considered a similar problem for single hop networks in [17]. The only difference is the constant vector $\boldsymbol{r}$ in place of the zero vector in the constraint equation. The vector $r$ arises due to the dependency of the communication rate of parent nodes on the rate of child nodes. We do not repeat the analysis here due to space limitations, but we summarize the main result in the following proposition.

Proposition 3. The maximum value of the objective function in (18) occurs either when one sensor transmits with probability one and all other sensors remain silent, or at a stationary point of the objective function, i.e. at $\boldsymbol{x}^{*}$ where $\nabla J\left(\boldsymbol{x}^{*}\right)=0$.

Since we may have multiple stationary points in the interior of the objective function domain, the proposition does not guarantee obtaining the global maximum. However, the proposition is still useful for the following reasons: 1) it avoids the case where the optimization algorithm may terminate at the local maximum $q_{i}=1, q_{j}=0$, while a better local maximum maybe at one of the stationary points, and 2) it provides information about the choice of the initial point for the optimization algorithm, where initial points near the corner points $q_{i}=1, q_{j}=0$ have to be avoided.

\section{PERformance Comparison}

We compare our design approach to the following two approaches.

Decoupled design. In this approach, each layer is designed separately. In the conventional slotted ALOHA, the MAC sublayer is designed to minimize the probability of collision, without regard to the QoI or CSI of each node. Minimum probability of collision occurs at $q_{k}=1 / N_{k}$, and consequently $P_{t}^{k}=p_{k} N_{k}$. The physical layer is designed to guarantee a minimum probability of successful packet transmission, $\lambda$. Using (2), we obtain:

$$
\bar{R}_{i}=W \log _{2}\left(1+10^{\left[0.1 \sigma_{c}^{i}\left(a_{i}-\Phi^{-1}[\lambda]\right)+\log N_{k}\right]}\right)
$$

and using (17), the deflection coefficient is given by:

$$
d^{2}=\frac{\tau}{b} J_{F C}, J_{k}=\frac{\lambda_{k}}{N_{k}}\left(1-\frac{1}{N_{k}}\right)^{N_{k}-1} \sum_{v \in \mathcal{C}_{k}}\left[J_{v}+\left(c_{v}-c_{k}\right) \bar{R}_{i}\right]
$$

where we assumed different value of $\lambda$ for each set of competing nodes. To make a fair comparison, we do not assume a pre-set value of $\lambda_{k}$. Rather, we optimize $\lambda_{k}$ values to yield the maximum deflection coefficient. The rate constraint in (18) reduces to:

$$
\lambda_{k} \leq \min _{v \in \mathcal{C}_{k}} \Phi\left[a_{v}-\left(\frac{10}{\sigma_{c}^{v}}\right)\left\{\log \left(2^{\frac{\bar{R}_{i}}{W}}-1\right)-\log N_{k}\right\}\right]
$$

Max Throughput Design. We follow the same hierarchical approach in defining and solving for the maximum throughput design. The throughput of any relay node $k$ is defined as:

$$
T_{k}=\sum_{v \in \mathcal{C}_{k}} \lambda_{v} \bar{R}_{v}
$$

The objective is to choose the design variables $q_{v}$ and $R_{v}$ to locally maximize the throughput. The constriant on the communication rate of node $v$ could be expressed in terms of its throughput as $\bar{R}_{v} \geq T_{v}$. The optimization problem could be formulated as:

$$
\max T_{k}=\sum_{v \in \mathcal{C}_{k}} \lambda_{v} \bar{R}_{v} \quad \text { s.t. } \bar{R}_{v} \geq T_{v}
$$




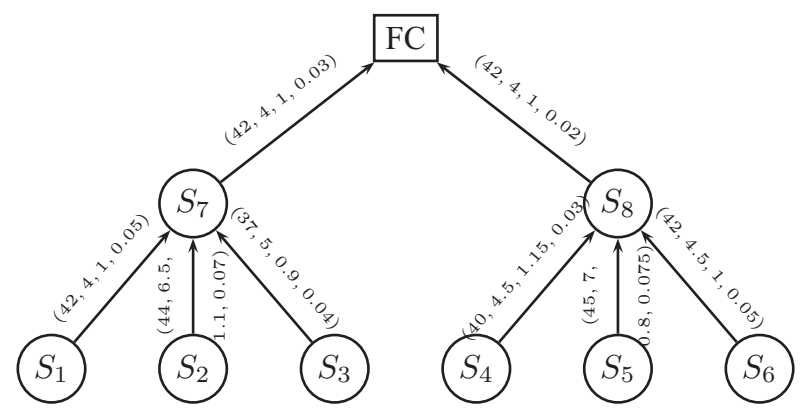

Figure 7. Detection tree network for the example problem. Labels on each edge represent $\mu_{c}, \sigma_{c}, e$ (in $\mathrm{mJ}$ ), and Signal to noise ratio, respectively.

where $T_{v}=0$ for leaf nodes. The optimal design variables could then be substituted back in (17) to evaluate the deflection coefficient.

\section{NUMERICAL EXAMPLE}

We consider the tree network in Figure 7, with system parameters as indicated. We use $W=2 \times 10^{3} \mathrm{~Hz}, N_{0}=$ $10^{-10}$, and $b=16$ bits. We use the interior-point algorithm to calculate the optimal solution in each case.

Figure (8) shows the deflection coefficient versus the delay for detection. The decoupled design approach shows the worst performance, since it does not take into account the application layer, in addition to the decoupling between the physical and MAC layers. The max throughput design outperforms the decoupled design, since it integrates both the physical and MAC layers. The cross-layer approach outperforms the two other approaches, by integrating the application layer (quality of the sensors) into the design process. This performance enhancement comes with no additional complexity since the optimization problem is very similar in the cross-layer and the max throughput design approaches. Therefore, for any value of the delay for detection, the detection performance is the highest for the cross-layer design. As another interpretation, to obtain the same detection performance from the other designs as in the cross-layer approach, the delay for detection has to increase significantly. Some values of the deflection coefficient may not be achievable by the other design approaches for any arbitrary value of the delay for detection.

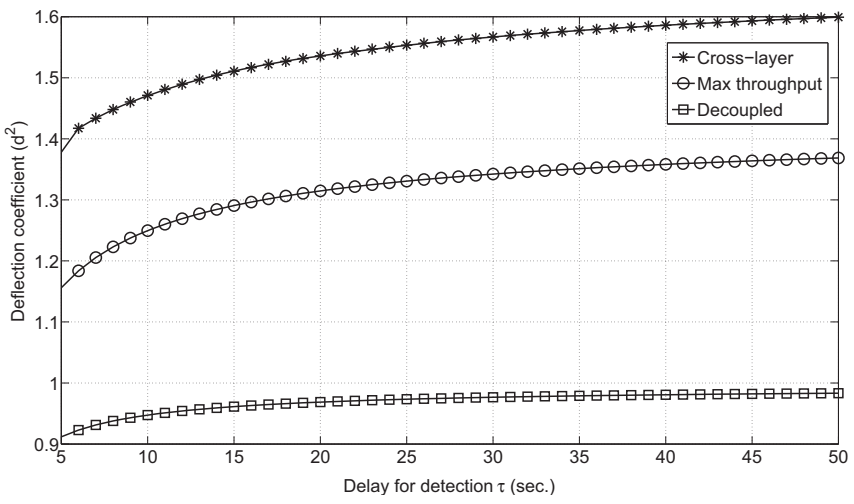

Figure 8. Deflection coefficient as it varies with the delay for detection.

\section{CONCLUSIONS}

We presented a cross-layer design approach for the Transmission Control Policy of tree wireless sensor networks deployed for detection applications. The TCP includes the transmission probabilities, communication rate, and power allocation for each sensor. The approach outperforms the max throughput and decoupled design approaches, for arbitrary delay for detection. The extension of this approach to mobile sensor networks and the inclusion of in-network information fusion are currently under research.

\section{REFERENCES}

[1] R. Viswanathan and P. Varshney, "Distributed detection with multiple sensors I. fundamentals," Proceedings of the IEEE, vol. 85, no. 1, pp. 54-63, Jan 1997.

[2] T. Duman and M. Salehi, "Decentralized detection over multiple-access channels," IEEE Transactions on Aerospace and Electronic Systems, vol. 34, no. 2, pp. 469-476, Apr 1998.

[3] M. Longo, T. Lookabaugh, and R. Gray, "Quantization for decentralized hypothesis testing under communication constraints," IEEE Transactions on Information Theory, vol. 36, no. 2, pp. 241-255, Mar 1990.

[4] V. V. Veeravalli and J.-F. Chamberland, "Detection in sensor networks," in Wireless Sensor Networks: Signal Processing and Communications Perspectives, A. Swami, Q. Zhao, Y.-W. Hong, and L. Tong, Eds. John Wiley \& Sons, Ltd, 2007, ch. 6, pp. 119-148.

[5] L. Liu and J.-F. Chamberland, "Cross-layer optimization and information assurance in decentralized detection over wireless sensor networks," in Fortieth Asilomar Conference on Signals, Systems and Computers, 2006. ACSSC '06., 29 2006-nov. 1 2006, pp. 271 -275.

[6] J. N. Tsitsiklis, "Decentralized detection," Advances in Signal Processing, vol. 2, pp. 297-344, 1993.

[7] Y. Yuan and M. Kam, "Distributed decision fusion with a randomaccess channel for sensor network applications," IEEE Transactions on Instrumentation and Measurement, vol. 53, no. 4, pp. 1339-1344, Aug. 2004.

[8] T.-Y. Chang, T.-C. Hsu, and P.-W. Hong, "Exploiting data-dependent transmission control and mac timing information for distributed detection in sensor networks," IEEE Transactions on Signal Processing, vol. 58, no. 3, pp. $1369-1382,2010$.

[9] Y. Yang, R. Blum, and B. Sadler, "Energy-efficient routing for signal detection in wireless sensor networks," IEEE Transactions on Signal Processing, vol. 57, no. 6, pp. 2050 -2063, June 2009

[10] Y. Sung, S. Misra, L. Tong, and A. Ephremides, "Cooperative routing for distributed detection in large sensor networks," Selected Areas in Communications, IEEE Journal on, vol. 25, no. 2, pp. $471-483$, feb. 2007.

[11] Z. M. Charbiwala, S. Zahedi, Y. Kim, Y. H. Cho, and M. B. Srivastava, "Toward quality of information aware rate control for sensor networks," Fourth International Workshop on Feedback Control Implementation and Design in Computing Systems and Networks, San Francisco, April 2009.

[12] Q. Zhao, A. Swami, and L. Tong, "The interplay between signal processing and networking in sensor networks," IEEE Signal Processing Magazine, vol. 23, no. 4, pp. 84-93, July 2006.

[13] M. Gastpar, "Uncoded transmission is exactly optimal for a simple gaussian sensor network," IEEE Transactions on Information Theory, vol. 54, no. 11, pp. 5247-5251, 2008.

[14] F. Zhao and L. Guibas, Wireless Sensor Networks: An Information Processing Approach. Morgan Kaufmann, 2004.

[15] M. Hata, "Empirical formula for propagation loss in land mobile radio services," IEEE Transactions on Vehicular Technology, vol. 29, no. 3, pp. 317 - 325, Aug. 1980.

[16] S. M. Kay, Fundamentals of Statistical Signal Processing, Volume 2: Detection Theory, ser. Prentice Hall Signal Processing Series, A. V. Oppenheim, Ed. Prentice Hall PTR, 1998.

[17] A. Tantawy, X. Koutsoukos, and G. Biswas, "Transmission Control Policy Design for Decentralized Detection in Sensor Networks," in IEEE International Conference on Distributed Computing in Sensor Systems, DCOSS 2011, Barcelona, Spain, June 27-29 2011. 